\title{
Environmental Science and Engineering
}

Vol: 1(1), 2022

REST Publisher; ISBN: 978-81-956353-2-0

Website: http://restpublisher.com/book-series/environmental-science-and-engineering/

\section{Hazard Identification and Risk Assessment in Glass Coater Plant}

*N. Dhinakaran, M. Balachandar

Department of Mechanical Engineering. (Industrial Safety Engineering), Sri Sai Ram Institute of Technology, Chennai, Tamil Nadu, India.

*Corresponding author Email: sitp20is08@sairamtap.edu.in

\begin{abstract}
In industrial arena, if any industry to be successful, it has to be safe, reliable, and sustainable in its operations. The industry has to identify the hazards and assess the associated risks and to bring the risks to tolerable level. Most of the accidents are caused by our latent or patent, visible or invisible, known or unknown, detectable or undetectable, intentional or unintentional unsafe acts, conditions and sequential events leading to accident. They explain rather the causation and not just the occurrence. Therefore, all should find the cause of accident and the appropriate measures for prevention. The zero-accident goal is an ideal goal, and everyone organization must try to achieve it by using all safety philosophy and technology. In reality, an industry and accidents are co-related and each industry is facing the accident problem, the nature, number and size may differ. It is this accident problem at the root which has generated the need of safety. Deaths, injuries and suffering are its direct results. Compensation, productionloss, time-loss and various costs-losses are indirect results. The injured worker, his family, factory and the nation, all are the sufferers. Accidents not resulting in human injuries, but resulting in property damage or money-loss are also accountable and undesired. Therefore, accident is always undesirable. That is why the accident problem has attracted attention worldwide. Industries are increasing to fulfil basic human needs food, clothing, home, employment or earning and requirements of goods, services and facilities. This brings industrial hazards of various types viz. mechanical, electrical, noise, vibration, chemical (fire, explosion, radiation, gas, dust, fumes, poisoning etc.) and many visible or invisible health hazards. It is the basic need to protect the human life and environment from all such hazards. Effective controls protect workers from workplace hazards, help avoid injuries, illnesses, and incidents, minimize or eliminate safety and health risks and help employers provide workers with safe and healthful working conditions. Only Risk Assessment can do it.
\end{abstract}

Keywords: Hazard, Risk, Incident, Risk Assessment, Risk Evaluation.

\section{Introduction}

To manage risk, hazards must first be identified, and then the risk should be evaluated and determined to be tolerate or not. The earlier in the life cycle that effective risk analysis is performed, the more cost effective the future safe operation of the process or activity is likely to be. The risk understanding developed from these studies forms the basis for establishing most of the other process safety management activities undertaken by the facility. An incorrect perception of risk at any point could lead to either inefficient use of limited resources or unknowing acceptance of risks exceeding the true tolerance of the company or the community. Hazard Identification and Risk Assessment (HIRA) is carried for identification of undesirable events that can lead to a hazard, the analysis of hazard of this undesirable event, that could occur and usually the estimation of its extent, magnitude and likelihood of harmful effects. It is widely accepted within industry in general that the various techniques of risk assessment contribute greatly toward improvements in the safety of complex operations and equipment. A Hazard Identification and Risk (HIRA) analysis is a systematic way to identify and analyse hazards to determine their scope, impact and the vulnerability of the built environment to such hazards and its purpose is to ensure that there is a formal process for hazard identification, risk assessment and control to effectively manage hazards that may occur within the workplaces. The objective of this work of hazards and risk analysis is to identify and analyse hazards, the event sequences leading to hazards and the risk associated with hazardous events. HIRA reviews may be performed at any stage in a project's life cycle - conceptual design, preliminary design, detailed design, construction, ongoing operation, decommissioning, or demolition. In general, the earlier that a hazard is identified (e.g., during conceptual design), the more cost-effectively it can be eliminated or managed. Studies performed during the early design stages are typically done at corporate or engineering offices. Studies performed once a process is near startup, during operation, or before decommissioning are typically done in a plant environment.

\section{Problem Identification}

Hazard identification is part of the process used to evaluate if any particular situation, item, thing, etc. may have the potential to cause harm. Hazard Identification is done at various locations of glass coater plant. Risk is calculated in every task performed at in each unit. Risk is calculated as the combination of Probability and Severity of hazard associated in each task. Risk is categorized as

- $\quad$ High Risk - Red Risk

- Medium Risk - Orange Risk

- $\quad$ Low Risk - Green Risk 
Red Risk activities which are serious in nature are identified work should not be started or continued until the impact has been reduced. If it is not possible to reduce impact even with unlimited resources, work has to remain prohibited. .Orange Risk Activities have moderate risk can be controlled by Considerable resources may have to be allocated to reduce the pact where the impact involves work in progress, urgent action should be taken. 5. Green Risk Activities are low risk activities in which general procedures are enough to control the risk. No additional controls are required. Consideration may be given to a more cost-effective solution or improvement that imposes no additional cost burden. Monitoring is required to ensure that the controls are maintained.

\section{Methodology}

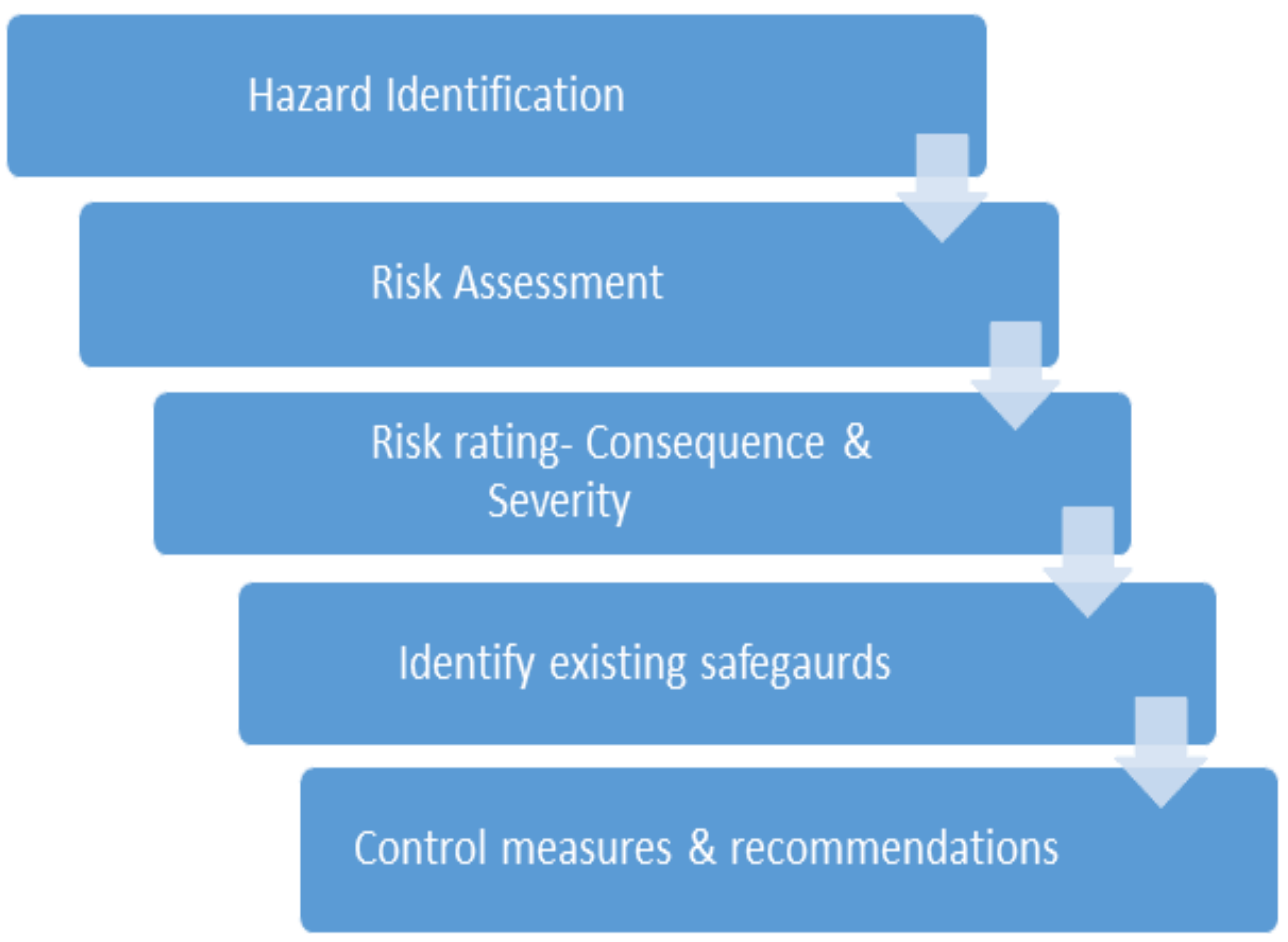

\section{Hazard Identification}

Identification of tasks carried out at the workplace (to identify all tasks so that they are all included in risk assessment). Consideration of tasks carried out at the workplace (evaluation of risks from the different tasks). Observation of work in progress (check that procedures are as laid down or predicted, and that there are no other risks arising). Consideration of patterns of work (to access exposure to hazards). Consideration of external factors that could affect the workplace (e.g., weather consideration for outdoor workers). Review of psychological, social and physical factors which might contribute to stress at work, how they interact together and with other factors in the workplace organization and environment. Consideration of organization to maintain conditions, including safeguards (e.g., that systems are in place to assess risks from new plant, materials and so on to update information on risks).

\section{Risk Assessment}

It is the quantitative evaluation of the likelihood of undesired events and their consequences being caused together and a value judgement concerning the significance of the results after comparing with set, legal or accepted values or standards. It is a judgement of significance or acceptability of risk identified by risk analysis and comparing against social, political or legal criteria, e.g., comparison of measured or calculated risk with the permissible safe limits and judgement regarding safety (whether the risk level is within or exceeding the safety limit) gives risk assessment. Objective of risk assessment are: 1. Identification of vulnerable zones (losses of persons and property) of the premises. 2. Estimation of hazard distances for the maximum credible accident (MCA) scenarios. 3. Suggestions for risk mitigation measures a delineation of approach to disaster management plan (DMP).

\section{Risk Rating}

Risk Rating is to find out probability of a hazard occurring and then an estimation of its consequence or effect and its severity. It is quantitative. For example, estimation of probability or frequency of possible explosion and its effect on persons and property in terms of deaths, injuries, house breakages 


\begin{tabular}{|c|c|c|c|c|c|}
\hline \multirow{2}{*}{ Severity } & \multicolumn{5}{|c|}{ Probability } \\
\hline & $\mathbf{1 0}$ & $\mathbf{8}$ & $\mathbf{6}$ & $\mathbf{4}$ & $\mathbf{1}$ \\
\hline & & & & & \\
\hline $\mathbf{1 0 0}$ & 1000 & 800 & 600 & 400 & 100 \\
\hline $\mathbf{4 0}$ & 400 & 320 & 240 & 160 & 40 \\
\hline $\mathbf{2 1}$ & 210 & 168 & 126 & 84 & 21 \\
\hline $\mathbf{8}$ & 80 & 64 & 48 & 32 & 8 \\
\hline $\mathbf{2}$ & 20 & 16 & 12 & 8 & 2 \\
\hline
\end{tabular}

7. Risk Control

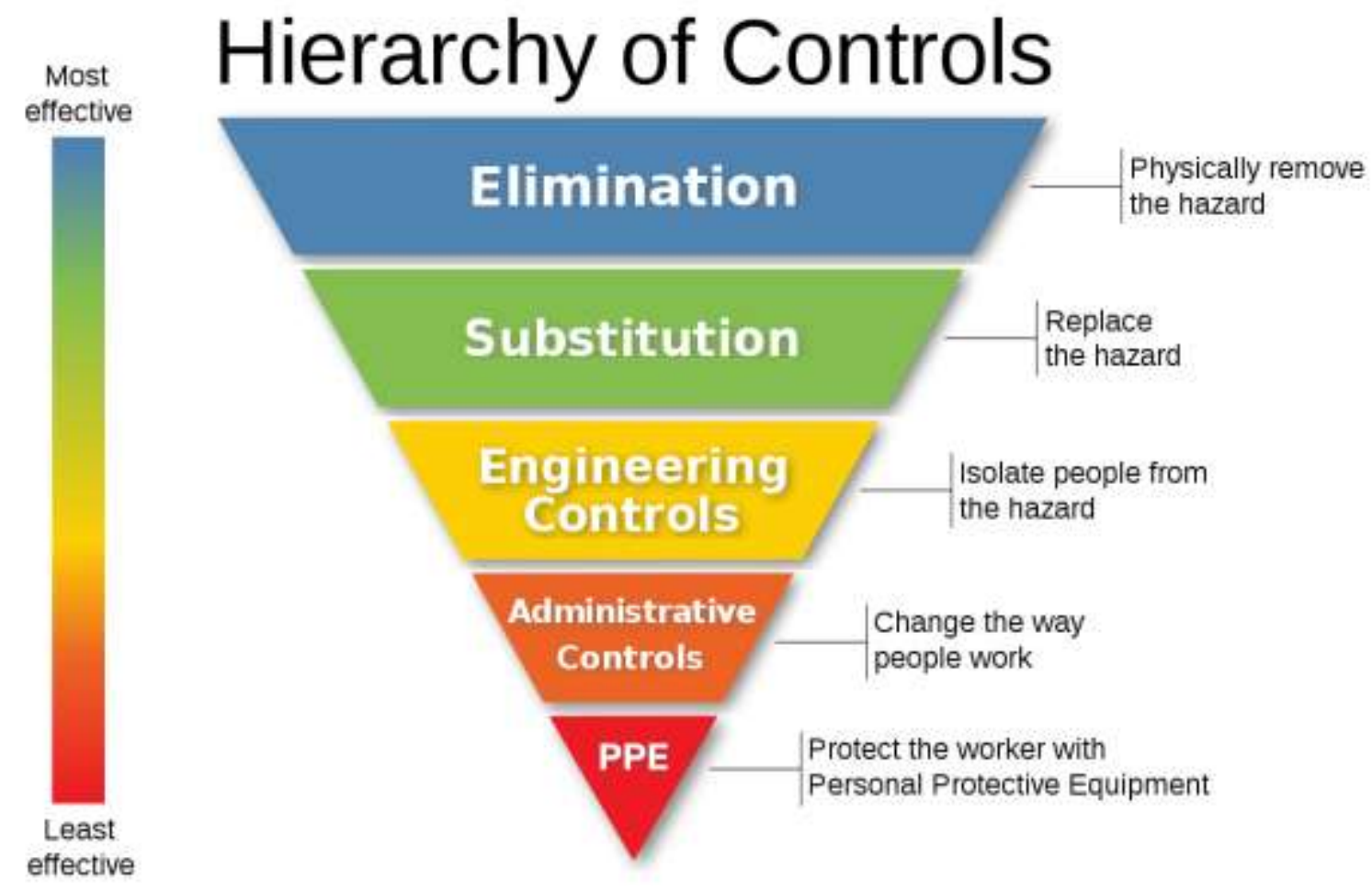

\section{Elimination and Substitution}

Elimination and substitution, while most effective at reducing hazards, also tend to be the most difficult to implement in an existing process. If the process is still at the design or development stage, elimination and substitution of hazards may be inexpensive and simple to implement. For an existing process, major changes in equipment and procedures may be required to eliminate or substitute for a hazard.

\section{Engineering Controls}

Engineering controls are favored over administrative and personal protective equipment (PPE) for controlling existing worker exposures in the workplace because they are designed to remove the hazard at the source, before it comes in contact with the worker. Well-designed engineering controls can be highly effective in protecting workers and will typically be independent of worker interactions to provide this high level of protection. The initial cost of engineering controls can be higher than the cost of administrative controls or PPE, but over the longer term, operating costs are frequently lower, and in some instances, can provide a cost savings in other areas of the process. 


\section{Administrative Controls and PPE}

Administrative controls and PPE are frequently used with existing processes where hazards are not particularly well controlled. Administrative controls and PPE programs may be relatively inexpensive to establish but, over the long term, can be very costly to sustain. These methods for protecting workers have also proven to be less effective than other measures, requiring significant effort by the affected workers.

\section{Conclusion}

On the basis of Hazard Identification and Risk Assessment conducted on glass coater plant revealed the risks associated with each of the task performed at the unit. Risks are classified as High, Medium, Low categories. Risk Control Measures are derived and mitigation plan with target date and responsibility of each risk is prepared and circulated to inter departments. Red Risk register is prepared based on high-risk activities and special focus is given to those activities. Review meetings are conducted based on this Hazard Identification and Risk Assessment report and roles and responsibilities are assigned to concerned department team faculties for implementing the risk control measures. Periodic review of risk control measures taken and its effectiveness should be monitored.

\section{References}

[1]. Risk Assessment: Theory, Methods, and Applications (2013) Marvin Rausand

[2]. Risk Assessment: Tools, Techniques, and Their Applications (2012) Cheryl A. Wilhelmsen and Lee T. Ostrom

[3]. The essentials of risk management (2008) Michel Crouhy

[4]. Risk Analysis: A Quantitative Guide(2005) David Vose

[5]. Donoghue, A. M., Occupational health hazards in Industries: an overview, http://occmed.oxfordjournals.org

[6]. Sharma, Vikrant, M. Ramachandran, Sathiyaraj Chinnasamy, and Vimala Saravanan. "A Review on Structural Equation Modeling and Its Classification."

[7]. Duijm, N. J., (2001), Hazard analysis of technologies for Industries, A90, pp. 123-135.

[8]. Chinnasamy, Sathiyaraj, M. Ramachandran, M. Amudha, and Kurinjimalar Ramu. "A Review on Hill Climbing Optimization Methodology." (2022).

[9]. Dziubinski, M., Fratczak, M. and Markowski, A. S., (2006), Journal of Loss Prevention in the Process Industries, Vol. 19, pp 399-408.

[10]. Venkateswaran, C., M. Ramachandran, Sathiyaraj Chinnasamy, Chinnasami Sivaji, and M. Amudha. "An Extensive Study on Gravitational Search Algorithm." (2022).

[11]. Khan, F. I. and Abbasi, S. A., (1998), Techniques and methodologies for risk analysis in chemical process industries, Journal of Loss Prevention in the Process Industries, Vol. 11, pp. 261-277. 